\title{
The Safety of Radiofrequency Ablation Using a Novel Temperature- Controlled Probe for the Treatment of Residual Intraductal Lesions after Endoscopic Papillectomy
}

\author{
Young Hoon Choi, Seung Bae Yoon, Jae Hyuck Chang, and In Seok Lee \\ Department of Internal Medicine, College of Medicine, The Catholic University of Korea, Seoul, Korea
}

See editorial on page 151.

\section{Article Info}

Received February 3, 2020

Revised April 17, 2020

Accepted April 23, 2020

Published online July 6, 2020

Corresponding Author

In Seok Lee

ORCID https://orcid.org/0000-0002-1127-1522

E-mail isle@catholic.ac.kr
Background/Aims: Treatment of residual intraductal lesions after endoscopic papillectomy for ampullary adenomas is relatively difficult. Few studies have been conducted using intraductal radiofrequency ablation (RFA) in the treatment of such lesions, and no study has aimed to reduce the side effects of excessive heat caused by RFA. Recently, a temperature-controlled RFA probe was developed to avoid excessive heat. This study aimed to investigate the safety of this new RFA probe in the treatment of intraductal lesions of ampullary adenoma.

Methods: Patients who received RFA for residual intraductal lesions after endoscopic papillectomy between November 2017 and June 2019 were retrospectively reviewed. A novel temperature-controlled probe (ELRA) was used for intraductal RFA, and clinical data including adverse events were collected.

Results: Ten patients were included in this study. Intraductal adenomas showed low-grade dysplasia in eight patients and high-grade dysplasia in two patients. The median diameter of intraductal adenomas was $9 \mathrm{~mm}$ (range, 5 to $10 \mathrm{~mm}$ ) in the common bile duct and $5 \mathrm{~mm}$ (range, 4 to $11 \mathrm{~mm}$ ) in the pancreatic duct. Adverse events occurred in three patients $(30.0 \%)$, of which two were mild pancreatitis and one was asymptomatic biliary stricture. Over a median follow-up period of 253 days, only one patient underwent additional surgery, as the remainder showed no adenomatous lesions on follow-up biopsies.

Conclusions: The new temperature-controlled RFA probe can be used with acceptable safety for the treatment of residual intraductal lesions after endoscopic papillectomy. Further evaluation through future prospective studies is needed. (Gut Liver 2021;15:307-314)

Key Words: Ampullary adenoma; Intraductal lesion; Radiofrequency ablation; Safety; Endoscopic papillectomy

\section{INTRODUCTION}

Ampullary adenoma is a rare disease with a prevalence of approximately $0.04 \%$ to $0.12 \%$ in autopsy studies, but it accounts for a large proportion of small intestine tumors. ${ }^{1,2}$ Since ampullary adenoma is a precancerous lesion that follows the adenoma-carcinoma sequence, removal with complete resection is the principle of treatment. ${ }^{3}$ Therefore, surgical resection, such as pancreaticoduodenectomy, has been widely used, but the associated morbidity and mortality is considerable. ${ }^{4,5}$ Another surgical method, transduodenal excision, has less morbidity but has a risk of recur- rence of nearly $30 \%{ }^{5,6}$ Due to this background, endoscopic papillectomy has recently become a preferred treatment for ampullary adenomas that lack evidence of an invasive cancer component. However, endoscopic papillectomy has the potential for incomplete resection, particularly if there is an intraductal extension of the ampullary adenoma. ${ }^{7}$ Thus, various methods, including radiofrequency (RF) ablation (RFA), have been studied to treat intraductal lesions of ampullary adenoma, but few such cases have been reported. ${ }^{8}$ Intraductal RFA is a method of necrosing a tumor using the heat from a RF electrode, and several studies have been conducted on patients with malignant biliary 
strictures. ${ }^{9}$ Previous studies have shown adverse events after intraductal RFA treatment, including ductal stricture, cholangitis, pancreatitis, and hemobilia, which are largely associated with tissue necrosis caused by excessive heat. ${ }^{10-12}$ To facilitate safer RFA, a temperature-controlled RF probe has recently been developed. This RF probe automatically adjusts the temperature of the RF electrode so that it does not rise above the initial set temperature, avoiding excessive heat. ${ }^{13}$ This probe has been tested for safety in a swine model of biliary stricture and recently in small prospective studies in patients with malignant biliary stricture. ${ }^{13,14}$ However, there have been no studies using only temperature-controlled RF probes for the treatment of intraductal extensions of ampullary adenoma. Therefore, the aim of this study was to determine the safety of RFA using a novel temperature-controlled RF probe for the treatment of residual intraductal lesions after endoscopic papillectomy.

\section{MATERIALS AND METHODS}

\section{Patients and data collection}

Patients with ampullary adenoma who underwent intraductal RFA for residual intraductal adenoma after endoscopic papillectomy from November 2017 to June 2019 at the Seoul Saint Mary's Hospital were included in this study. Inclusion criterion was the presence of intraductal adenoma confirmed by intraductal biopsy in distal common bile duct or pancreatic duct. Exclusion criteria were patients who did not consent to the RFA procedure, presence of resectable periampullary cancer, acquired or congenital coagulopathy, prior gastric surgery preventing the endoscopic retrograde cholangiopancreatography (ERCP) procedure (e.g., Billroth II or Roux-en-Y), less than 18 years old, and pregnancy. We collected patient demographic data, characteristics of the ampullary adenoma, endoscopy reports of intraductal lesions after endoscopic papillectomy, details of RFA treatment, follow-up duration, pathologic results at last follow-up, and adverse event. Adverse events were evaluated based on the American Society for Gastrointestinal Endoscopy lexicon. ${ }^{15}$ This study protocol was based on the Declaration of Helsinki and approved by the Institutional Review Board of Seoul Saint Mary's Hospital (IRB number: KC19RESI0540). Written informed consent for RFA and follow-up was obtained from all patients prior to the procedure.

\section{Study procedure and outcome measurements}

All ERCP and intraductal RFA procedures were performed by two expert endoscopists. After initial endoscopic papillectomy and at follow-up, the intraductal

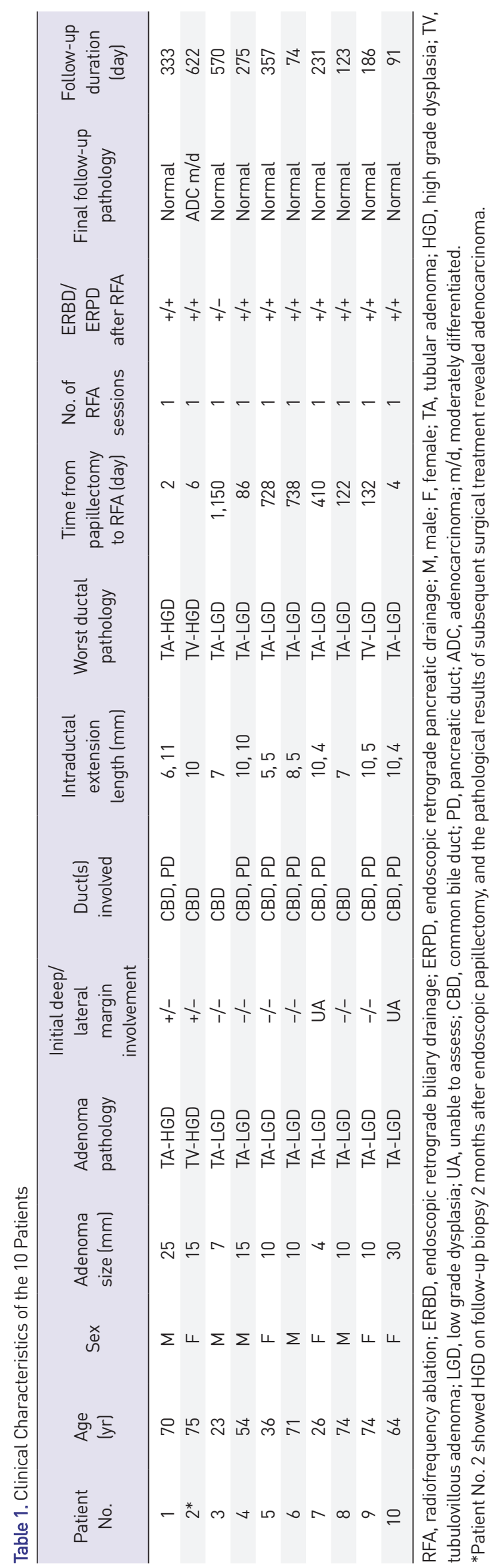


extension of the ampullary adenoma was determined using cholangiogram or pancreatogram through ERCP, or through direct observation of the bile duct or pancreatic duct orifice via duodenoscope. Prior to the procedure, second generation cephalosporin antibiotics were administered prophylactically. When a recurrent adenomatous lesion involved in both the duodenal wall and the bile duct or pancreatic duct, snare resection of the duodenal lesion was performed first, followed by RFA in the same session. On the other hand, if there was no lesion that could be excised with snare on the duodenal side, RFA was performed without snare resection.

A RFA catheter (ELRA ${ }^{\mathrm{TM}}$, STARmed, Goyang, Korea) and RF generator system (VIVA Combo ${ }^{\mathrm{TM}}$, STARmed) were used for intraductal RFA procedures. The RFA catheter used in our study was $7 \mathrm{~F}$ large and $175-\mathrm{cm}$ long with bipolar electrodes of 4-mm width and 11-mm length. The RFA catheter was inserted into the biliary or pancreatic duct through a 0.025 - or 0.035 -inch guidewire. If lesions were involved in both ducts, RFA was performed in the biliary and pancreatic ducts, respectively. The RF generator system used in this study provided a temperature mode to maintain the set temperature during the procedure to prevent excessive heating. The generator was set at a maximum temperature of $80^{\circ} \mathrm{C}$ and a power of $7 \mathrm{~W}$ with reference to previous studies. ${ }^{8,13,14}$ Intraductal RFA was performed within 90 seconds while observing the degree of whitish color change due to heat around the duct orifice. After RFA, both biliary stents with 7-F or 10-F diameter (Cotton-Leung or Zimmon or ST-2 Soehendra Tannenbaum biliary stent; Cook Medical, Winston-Salem, NC, USA) and pancreatic stents with 5-F or 7-F diameter (Geenen pancreatic stent; Cook Medical) were placed in all possible cases for preventing cholangitis and pancreatitis. Follow-up endoscopy was performed 2 days after RFA to evaluate the healing status of the RFA site, and acute complications such as bleeding. Biliary and pancreatic plastic stents were removed by follow-up endoscopy 1 to 2 months after RFA. Subsequent follow-up endoscopies were performed every 3 to 6 months within 1 year from RFA, and every 6 months to 1 year thereafter. At all followup endoscopy, biopsy was performed at the margin of the RFA site to assess the recurrence of adenoma. Computed tomography scans were performed around 6 months after RFA to evaluate duct stricture. After that, if clinically suspected of duct stricture, imaging tests, such as computed tomography or magnetic resonance cholangiopancreatography, were performed at the physician's discretion.

The primary endpoint was the incidence of adverse events after intraductal RFA. The secondary endpoint was the rate of successful endoscopic treatment, which was defined as the absence of adenomatous tissue in the followup biopsy.

\section{Statistical analysis}

Categorical variables are presented as a number, and continuous variables are shown as the median and range. Statistical analyses were performed using SPSS software, version 24.0 (IBM Corp., Armonk, NY, USA).

\section{RESULTS}

During the study period, a total of 49 patients underwent endoscopic papillectomy. Among these patients, 10 patients were confirmed residual intraductal lesion by intraductal biopsy and treated with endoscopic RFA. The clinical data, including demographics, initial adenoma size and pathology, duct involvement of the residual adenoma, and follow-up data, are shown in Table 1. Fig. 1 shows representative cholangiopancreatograms during RFA treatment. Figs 2 and 3 show representative endoscopic images
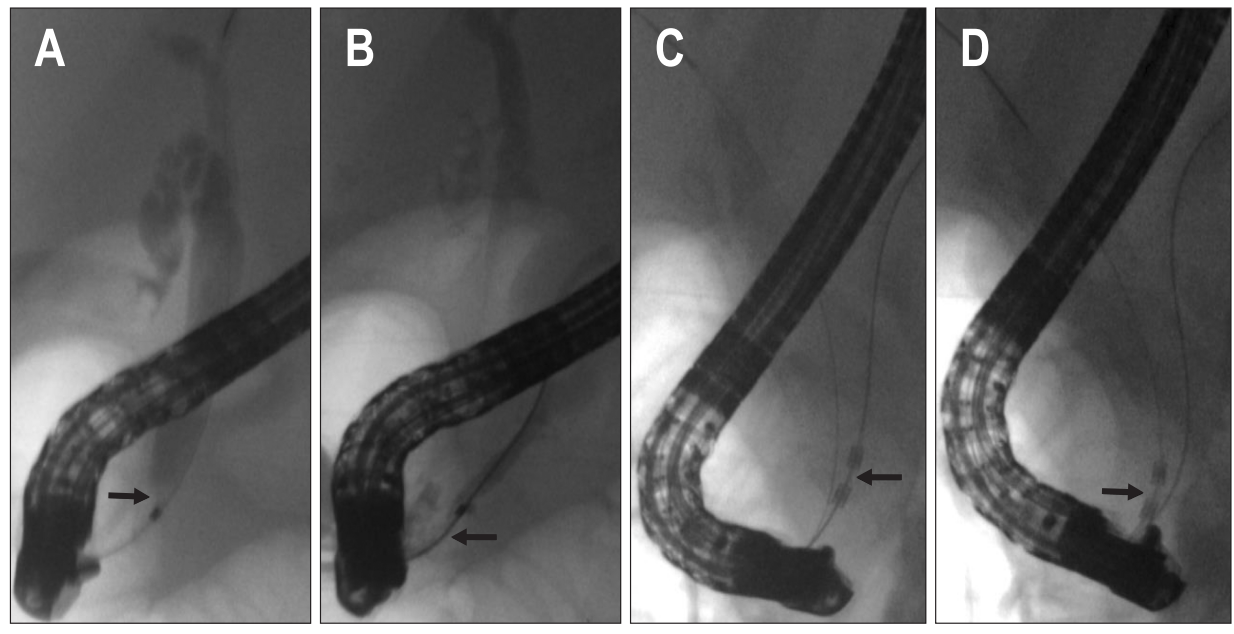

Fig. 1. Cholangiopancreatograms showing the intraductal extension of the ampullary adenoma into the bile duct (A) and pancreatic duct (B). The radiofrequency ablation probe is located in the pancreatic duct (C) and bile duct (D) (patient No. 1). 

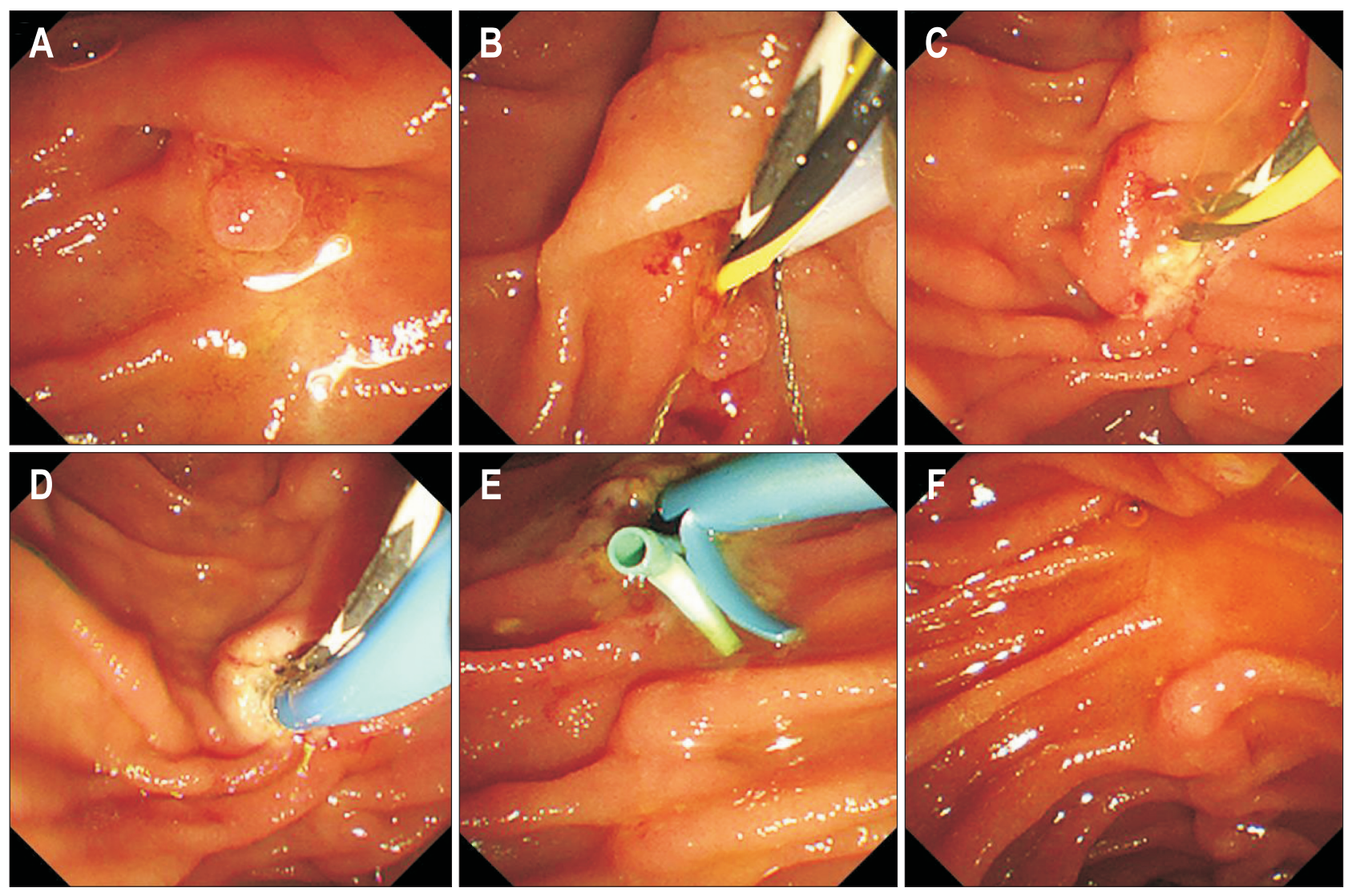

Fig. 2. Endoscopic images showing radiofrequency ablation (RFA) treatment after snare resection. The adenomatous lesion on the duodenal wall (A) was resected with a snare (B, C). Intraductal RFA of the bile duct (D) with whitish color change around the duct orifice. Placement of biliary and pancreatic plastic stents after RFA (E). The last follow-up endoscopic image showing no recurrence (F) (patient No. 8).

with or without snare resection before RFA treatment, respectively.

Ampullary adenomas were detected through screening esophagogastroduodenoscopy in five patients and through surveillance esophagogastroduodenoscopy for extracolonic malignancy in three patients known as familial adenomatous polyposis. The two remaining cases were found on esophagogastroduodenoscopy performed on the suspicion of ampullary lesions based on abdominal computed tomography.

Three patients with recurrent duodenal and intraductal adenomatous lesions underwent snare resection of duodenal lesion prior to the RFA procedure. The size of the duodenal lesions in these patients were all around $5 \mathrm{~mm}$. Three patients underwent intraductal RFA for a residual intraductal lesion within 1 week after endoscopic papillectomy, and the remaining seven patients underwent RFA for a residual intraductal lesion at least 3 months after the initial endoscopic papillectomy. For those seven patients, the median time from endoscopic papillectomy to RFA was 13.7 months (range, 2.9 to 38.3 months). All 10 patients underwent intraductal RFA for the common bile duct with a median RFA delivery time of 65 seconds (range, 45 to 90 seconds), and seven of the 10 patients also underwent RFA for the pancreatic duct with a median RFA delivery time of 30 seconds (range, 15 to 75 seconds). After RFA, biliary stents were inserted in all 10 patients, and pancreatic stents were inserted in nine of the 10 patients including all patients who received RFA for the pancreatic duct.

The median follow-up duration was 253 days (range, 74 to 622 days). Nine of the 10 patients had no evidence of adenomatous lesion in the last follow-up pathology. The remaining patient had high-grade dysplasia on the followup pathologic examination performed approximately 2 months after RFA. The patient underwent a pylorus-preserving pancreaticoduodenectomy, and the dysplasia was confirmed as adenocarcinoma in the surgical specimen pathology. Patient follow-up results are summarized in Fig. 4.

Adverse events occurred in three patients. Two patients had mild pancreatitis. Both had prophylactic pancreatic stents, and improved with conservative management. The other patient had asymptomatic biliary stricture confirmed through ERCP performed about 5 months after the intra- 

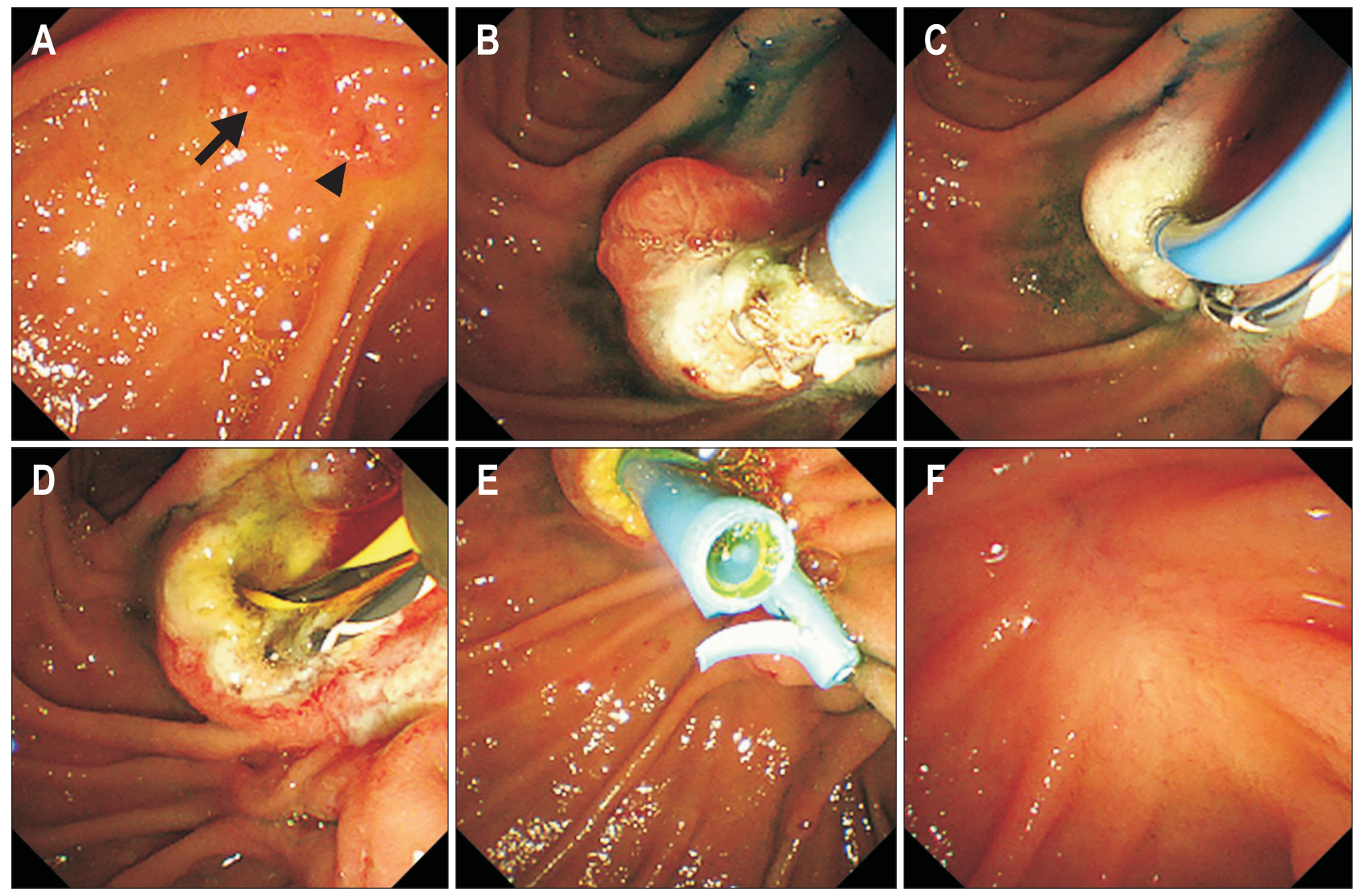

Fig. 3. Endoscopic images showing radiofrequency ablation (RFA) treatment without snare resection. Adenomatous lesions around the duct (biliary, arrow; pancreatic, arrowhead) orifices (A). Intraductal RFA of the pancreatic duct (B) and bile duct (C) with the characteristic whitish color change apparent around the duct orifice. Appearance after RFA (D). Placement of plastic biliary and pancreatic stents after RFA (E). The last follow-up endoscopic image, showing no recurrence (F) (patient No. 5).

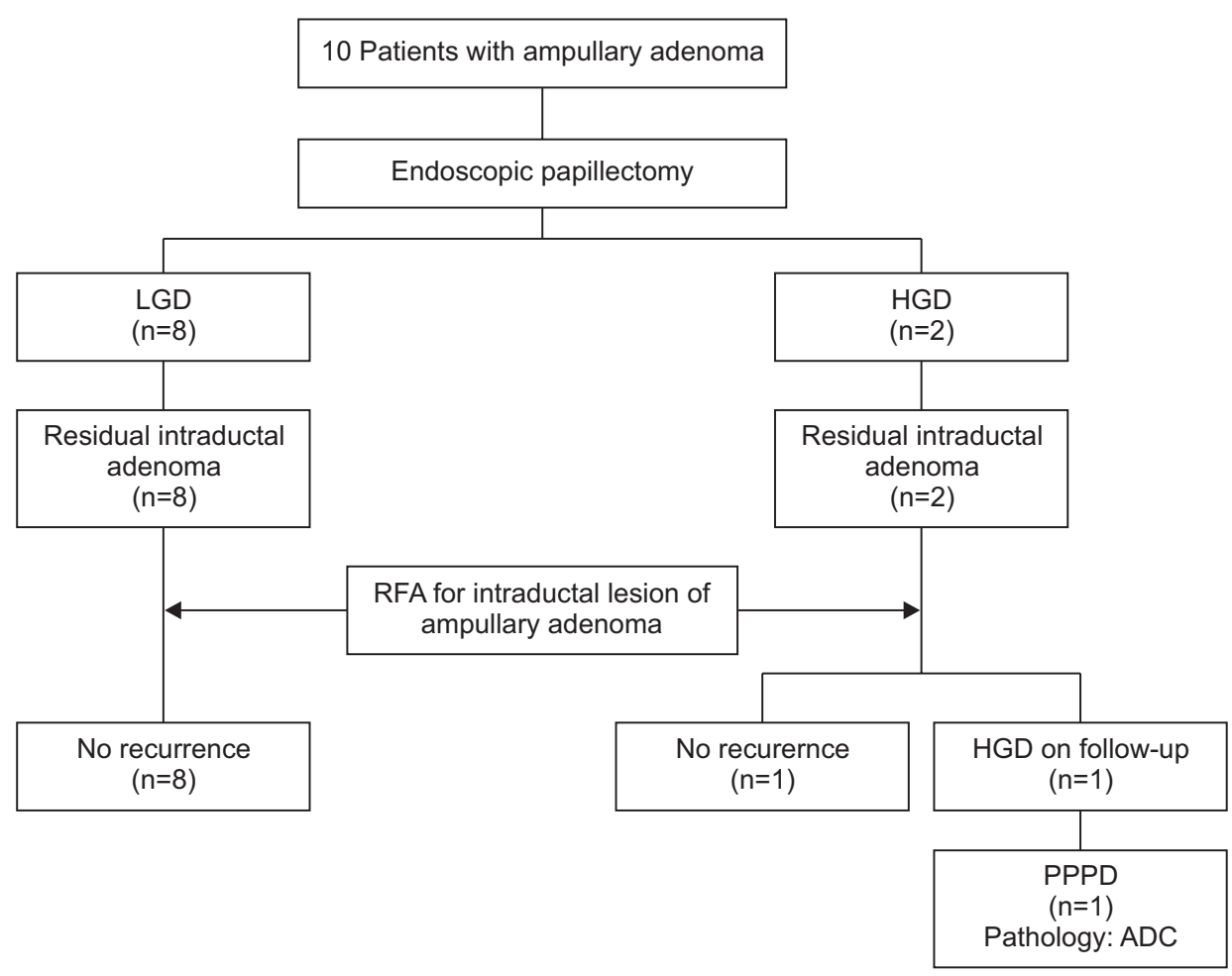

Fig. 4. Flowchart summarizing overall results.

LGD, low grade dysplasia; HGD, high grade dysplasia; RFA, radiofrequency ablation; PPPD, pylorus preserving pancreaticoduodenectomy; ADC, adenocarcinoma. 
Table 2. Adverse Events

\begin{tabular}{lc}
\hline \multicolumn{1}{c}{ Characteristics } & No. (patient No.) \\
\hline Pancreatitis & 2 (patient No. 5, 7) \\
Cholangitis & 0 \\
Bleeding & 0 \\
Perforation & 0 \\
Nonsymptomatic biliary stricture de- & 1 (patient No. 1) \\
tected at ERCP & \\
\hline
\end{tabular}

ERCP, endoscopic retrograde cholangiopancreatography.

ductal RFA. This patient underwent bougie dilation for the biliary stricture and experienced no subsequent biliary complications (Table 2).

\section{DISCUSSION}

In the treatment of ampullary adenoma, the relatively less-invasive endoscopic papillectomy is currently the preferred treatment compared to surgical treatments with significant morbidity or mortality. The recurrence rate after endoscopic papillectomy varies from study to study, but a rate up to $33 \%$ has been reported. ${ }^{16}$ To reduce recurrence after endoscopic snare papillectomy, additional treatments, such as ablation using argon plasma coagulation, can be performed, but their efficiency is low. ${ }^{17,18}$ In addition, complete endoscopic treatment is difficult, especially in cases of ampullary adenoma with an intraductal extension of the bile duct or pancreatic duct. ${ }^{7}$ The difficulty in treating these intraductal lesions is due to the difficulty in accessing the ductal structures using conventional devices for endoscopic treatment of ampullary adenoma such as snares or argon plasma coagulation.

RFA is a treatment for killing cancer cells by using the heat generated by electrodes and is currently used for the treatment of various solid tumors including hepatocellular carcinoma. ${ }^{19,20}$ Since the electrodes used in RFA can be inserted into ductal structures using a guide wire during ERCP, RFA is particularly useful for the treatment of intraductal lesions such as malignant biliary strictures. ${ }^{21} \mathrm{RFA}$ is also being introduced into the treatment of intraductal extensions of ampullary adenoma due to its aforementioned features. Mehendiratta and Desilets ${ }^{22}$ reported a case of eradication of residual adenoma using RFA after endoscopic papillectomy. In addition, Valente et al. ${ }^{23}$ reported three cases of successful treatment via RFA in patients with ampullary adenoma who either refused or could not receive surgical treatment. Recently, two small studies of RFA after endoscopic papillectomy in 14 patients and 20 patients, respectively, were published. ${ }^{8,12}$ In those two studies, the rate of successful endoscopic therapy was $70 \%$ to $92 \%$, which does not appear to be notably different from the previously reported successful treatment rate of endoscopic papillectomy. ${ }^{18}$ However, those studies only included patients with intraductal lesions that were relatively difficult to treat; therefore, the treatment success rate of those studies can be seen as relatively high given the circumstances. Our study also showed a successful endoscopic treatment rate of $90 \%$, similar to the previous studies.

While the success rate of treatment was relatively high, the rate of adverse events after RFA was as high as $40 \%$ to $43 \%$ in both of the previous two studies. ${ }^{8,12}$ These adverse event rates are higher than those after endobiliary RFA in malignant biliary stricture. ${ }^{21,24}$ Compared with RFA for malignant biliary stricture, RFA for ampullary adenomas is performed at the ampullary orifice area and can be more damaging to surrounding normal tissues, which can increase the risk of adverse events. As these adverse events are often caused by excessive heat, a temperaturecontrolled RF probe has been developed and studied in patients with malignant biliary stricture. ${ }^{14}$ However, in the case of ampullary adenoma, there have been no previous studies using only a temperature-controlled RF probe. In this study, we aimed to evaluate the safety of a temperaturecontrolled RF probe for the treatment of intraductal extensions of ampullary adenoma. The incidence of adverse events, including pancreatitis and asymptomatic biliary stricture, was $30 \%$ in our study. Although it is difficult to make accurate comparisons due to the low number of subjects, the rate of adverse events in our study was somewhat lower than the $40 \%$ to $43 \%$ of previous studies. ${ }^{8,12}$ This lower rate of adverse events may be attributed to the avoidance of excessive heating using a temperature-controlled $\mathrm{RF}$ probe, but future prospective studies are required for validation. In addition to temperature control, the RFA device in this study also featured an RF electrode length of $11 \mathrm{~mm}$, which is shorter than the length used in previous studies. Because the treatment success rate was similar to that of previous studies, it is possible that the ablation of the unnecessary region was minimized by the shorter electrodes, leading to fewer adverse events. The fact that the length of the stricture was significantly longer when using longer probes in an endobiliary RFA animal study supports this inference. ${ }^{25}$ In some cases, longer RFA probes may be required for complete treatment, but shorter probes are desirable when possible. In other words, it is inferred that patients with short intraductal lesions that can use short RFA probe may benefit the most from RFA treatment. The depth and width of the RFA area, which is closely related to the treatment efficiency and the occurrence of adverse events, is determined by a combination of power, time, and temperature. ${ }^{26}$ Therefore, further research is needed on the 
optimal settings for these parameters for the treatment of ampullary adenomas, keeping in mind that further adjustments will be needed depending on the specific lesion.

There are several limitations in our study. First, this study is based on retrospective data derived from a small number of patients. Second, the follow-up period was relatively short, and recurrence during follow-up was evaluated by endoscopic findings and biopsy mainly. In particular, the proximal margin of RFA could not be properly evaluated by biopsy because follow-up endoscopic examinations were performed in the outpatient department. Therefore, in next study, it would be ideal to evaluate the recurrence of intraductal lesion with sufficient follow-up period using biopsy through cholangioscopy, and endoscopic ultrasound. In spite of these limitations, this is the first study that showed acceptable safety of a new temperature-controlled RF probe for the treatment of ampullary adenoma after endoscopic papillectomy.

In conclusion, RFA using a temperature-controlled RF probe showed acceptable safety without serious side effects in 10 patients with residual intraductal lesions after endoscopic papillectomy. Avoiding excessive heat using temperature-controlled RFA is expected to reduce adverse events, and future prospective studies are needed to confirm whether this method is significantly safer than other RFA methods.

\section{CONFLICTS OF INTEREST}

No potential conflict of interest relevant to this article was reported.

\section{AUTHOR CONTRIBUTIONS}

Study concept and design: I.S.L., Y.H.C. Data acquisition: Y.H.C., S.B.Y. Data analysis and interpretation: J.H.C., S.B.Y. Drafting of the manuscript: Y.H.C. Critical revision of the manuscript: S.B.Y., J.H.C., I.S.L.

\section{ORCID}

Young Hoon Choi

https://orcid.org/0000-0002-2633-1401

Seung Bae Yoon https://orcid.org/0000-0002-6119-7236

Jae Hyuck Chang https://orcid.org/0000-0003-1180-2693

In Seok Lee https://orcid.org/0000-0002-1127-1522

\section{REFERENCES}

1. Chini P, Draganov PV. Diagnosis and management of ampullary adenoma: the expanding role of endoscopy. World J Gastrointest Endosc 2011;3:241-247.

2. Baker HL, Caldwell DW. Lesions of the ampulla of Vater. Surgery 1947;21:523-531.

3. Stolte M, Pscherer C. Adenoma-carcinoma sequence in the papilla of Vater. Scand J Gastroenterol 1996;31:376-382.

4. Di Giorgio A, Alfieri S, Rotondi F, et al. Pancreatoduodenectomy for tumors of Vater's ampulla: report on 94 consecutive patients. World J Surg 2005;29:513-518.

5. Martin JA, Haber GB. Ampullary adenoma: clinical manifestations, diagnosis, and treatment. Gastrointest Endosc Clin N Am 2003;13:649-669.

6. Farnell MB, Sakorafas GH, Sarr MG, et al. Villous tumors of the duodenum: reappraisal of local vs. extended resection. J Gastrointest Surg 2000;4:13-21.

7. Bohnacker S, Seitz U, Nguyen D, et al. Endoscopic resection of benign tumors of the duodenal papilla without and with intraductal growth. Gastrointest Endosc 2005;62:551-560.

8. Rustagi T, Irani S, Reddy DN, et al. Radiofrequency ablation for intraductal extension of ampullary neoplasms. Gastrointest Endosc 2017;86:170-176.

9. Larghi A, Rimbaș M, Tringali A, Boškoski I, Rizzatti G, Costamagna G. Endoscopic radiofrequency biliary ablation treatment: a comprehensive review. Dig Endosc 2019;31:245255.

10. Sharaiha RZ, Sethi A, Weaver KR, et al. Impact of radiofrequency ablation on malignant biliary strictures: results of a collaborative registry. Dig Dis Sci 2015;60:2164-2169.

11. Yang J, Wang J, Zhou H, et al. Efficacy and safety of endoscopic radiofrequency ablation for unresectable extrahepatic cholangiocarcinoma: a randomized trial. Endoscopy 2018;50:751-760.

12. Camus M, Napoléon B, Vienne A, et al. Efficacy and safety of endobiliary radiofrequency ablation for the eradication of residual neoplasia after endoscopic papillectomy: a multicenter prospective study. Gastrointest Endosc 2018;88:511518.

13. Cho JH, Lee KH, Kim JM, Kim YS, Lee DH, Jeong S. Safety and effectiveness of endobiliary radiofrequency ablation according to the different power and target temperature in a swine model. J Gastroenterol Hepatol 2017;32:521-526.

14. Lee YN, Jeong S, Choi HJ, et al. The safety of newly developed automatic temperature-controlled endobiliary radiofrequency ablation system for malignant biliary strictures: a prospective multicenter study. J Gastroenterol Hepatol 2019;34:1454-1459.

15. Cotton PB, Eisen GM, Aabakken L, et al. A lexicon for endoscopic adverse events: report of an ASGE workshop. Gastro- 
intest Endosc 2010;71:446-454.

16. Han J, Kim MH. Endoscopic papillectomy for adenomas of the major duodenal papilla (with video). Gastrointest Endosc 2006;63:292-301.

17. Nam K, Song TJ, Kim RE, et al. Usefulness of argon plasma coagulation ablation subsequent to endoscopic snare papillectomy for ampullary adenoma. Dig Endosc 2018;30:485492.

18. ASGE Standards of Practice Committee, Chathadi KV, Khashab MA, et al. The role of endoscopy in ampullary and duodenal adenomas. Gastrointest Endosc 2015;82:773-781.

19. Kim YS, Lim HK, Rhim H, et al. Ten-year outcomes of percutaneous radiofrequency ablation as first-line therapy of early hepatocellular carcinoma: analysis of prognostic factors. J Hepatol 2013;58:89-97.

20. Shah DR, Green S, Elliot A, McGahan JP, Khatri VP. Current oncologic applications of radiofrequency ablation therapies. World J Gastrointest Oncol 2013;5:71-80.

21. Sofi AA, Khan MA, Das A, et al. Radiofrequency ablation combined with biliary stent placement versus stent placement alone for malignant biliary strictures: a systematic review and meta-analysis. Gastrointest Endosc 2018;87:944951.

22. Mehendiratta V, Desilets DJ. Use of radiofrequency ablation probe for eradication of residual adenoma after ampullectomy. Gastrointest Endosc 2015;81:1055-1056.

23. Valente R, Urban O, Del Chiaro M, et al. ERCP-directed radiofrequency ablation of ampullary adenomas: a knifesparing alternative in patients unfit for surgery. Endoscopy 2015;47 Suppl 1 UCTN:E515-E516.

24. Zheng X, Bo ZY, Wan W, et al. Endoscopic radiofrequency ablation may be preferable in the management of malignant biliary obstruction: a systematic review and meta-analysis. J Dig Dis 2016;17:716-724.

25. Cho JH, Jeong S, Kim EJ, Kim JM, Kim YS, Lee DH. Longterm results of temperature-controlled endobiliary radiofrequency ablation in a normal swine model. Gastrointest Endosc 2018;87:1147-1150.

26. Borne RT, Sauer WH, Zipse MM, Zheng L, Tzou W, Nguyen DT. Longer duration versus increasing power during radiofrequency ablation yields different ablation lesion characteristics. JACC Clin Electrophysiol 2018;4:902-908. 